\title{
Incorporación de tecnologías en la enseñanza de las matemáticas Actitudes del estudiantado universitario
}

Incorporating technologies in math teaching:

Attitudes of higher education students
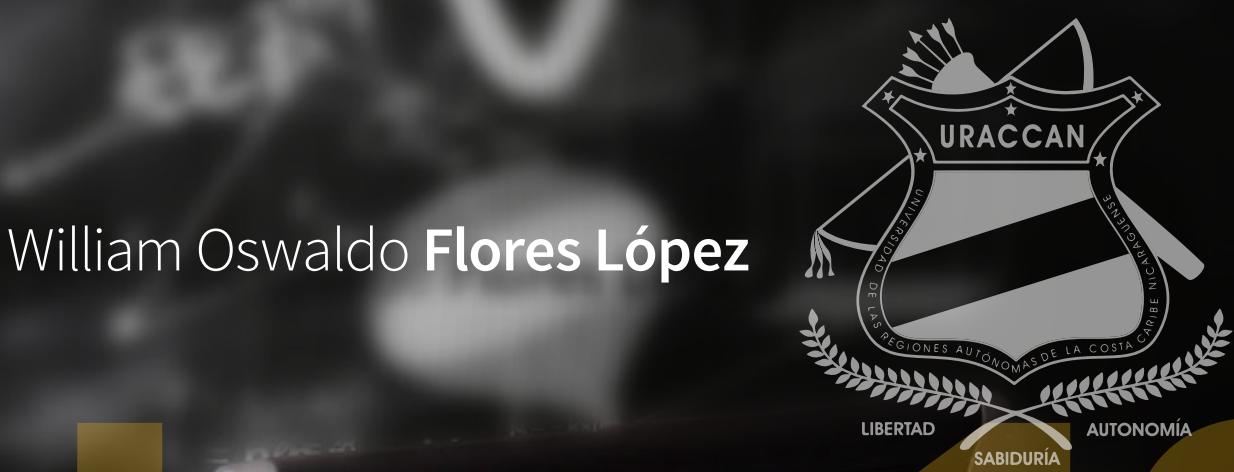

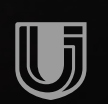

IBEROAMERICANA CORPORACIÓN UNIVERSITARIA
HORIZONTES

PEDAGÓGICOS

ISSN-I: 0123-8264 | e-ISSN: 2500-705X 


\section{HORIZONTES PEDAGÓGICOS}

ID:

0123-8264.hop.19103

Title: Incorporating technologies in math teaching

Subtitle: Attitudes of higher education students

Título: Incorporación de tecnologías en la enseñanza de las matemáticas

Subtítulo: Actitudes del estudiantado universitario

Alt Title / Título alternativo:

[en]: $\quad$ University student attitudes to the incorporation of technologies in teaching mathematics

[es]:

\section{Actitudes del estudiantado} universitario ante la incorporación de tecnologías en la enseñanza de las matemáticas

Author (s) / Autor (es):

Flores López

Keywords / Palabras Clave:

[en]: attitude; technology; mathematics; teaching; assessment

[es]: $\quad$ actitud; tecnología; matemáticas enseñanza; evaluación

Proyecto / Project:

Actitudes del estudiantado ante la incorporación de tecnologías en la enseñanza-aprendizaje de la estadística-matemática

Submited: 2016-11-08

Acepted: 2017-04-06

\section{Resumen}

En esta investigación se han analizado las actitudes del estudiantado universitario ante la incorporación de tecnologías en la enseñanza de las matemáticas. Se trata de un estudio cuantitativo sustentado en un diseño descriptivo donde se suministró una escala de actitud a una muestra de 106 estudiantes universitarios de una Universidad de la Costa Caribe Nicaragüense. Los resultados indican que el estudiantado siente gusto y motivación al tener experiencias matemáticas con tecnologías; que hombres y mujeres provenientes de los grados en informática, ingeniería civil y matemática poseen las mismas actitudes; y que la edad entre 17 y 20 años es determinante por el gusto y motivación que siente el estudiantado en aprender matemáticas con tecnologías. Se concluye que incorporar tecnología en la enseñanza de las matemáticas contribuye a la actitud del estudiantado hacia las matemáticas.

\section{Abstract}

With the goal of analyzes the attitudes of the higher education students, in the adoption of technologies in the Math teaching process, this research does a quantitative study, based on a descriptive design. This provides an attitude scale, with a sample of 106 students of the Universidad de las Regiones Autonomas de la Costa Caribe Nicaraguense; the results show how the students increase their feelings and motivations when they obtain better experiences in math learning with the use of technologies to improve this. Moreover, at independent of the genre, the students of engineering programs as computing or civil, and the same math program shows in the evidence a same or similar attitude. In the end, the students feel better and motivated in the

math learning with the use of technologies; especially in ages surround 17 to 20 . Therefore, when technologies are applied to improve the math teaching, these efforts produces more motivations and other favorable attitudes toward to math learning.
DrWilliam Oswaldo Flores López, MSc

Research ID: L-6857-2015

ORCID: $\quad$ 0000-0002-1016-1620

\section{Source I Filiacion:}

Universidad de las Regiones Autónomas de la Costa Caribe Nicaragüense

BIO:

Doctor en Educación, Universidad de Deusto

Máster en Investigación en didácticas de las Ciencias Experimentales y Matemáticas Profesor de Matemáticas, Universidad de las Regiones Autónomas de la Costa Caribe Nicaragüense

\section{City I Ciudad:}

Nueva Guinea [ni]

e-mail:

william.flores@uraccan.edu.ni

Citar como:

Flores, W. O. (2017). Incorporación de tecnologías en la enseñanza de las matemáticas: Actitudes del estudiantado universitario. Horizontes Pedagógicos issn-l:0123-8264, 19 (1), 21-30

Obtenido de: https://revistas.iberoamericana.edu.co/index.php/rhpedagogicos/article/view/1101 


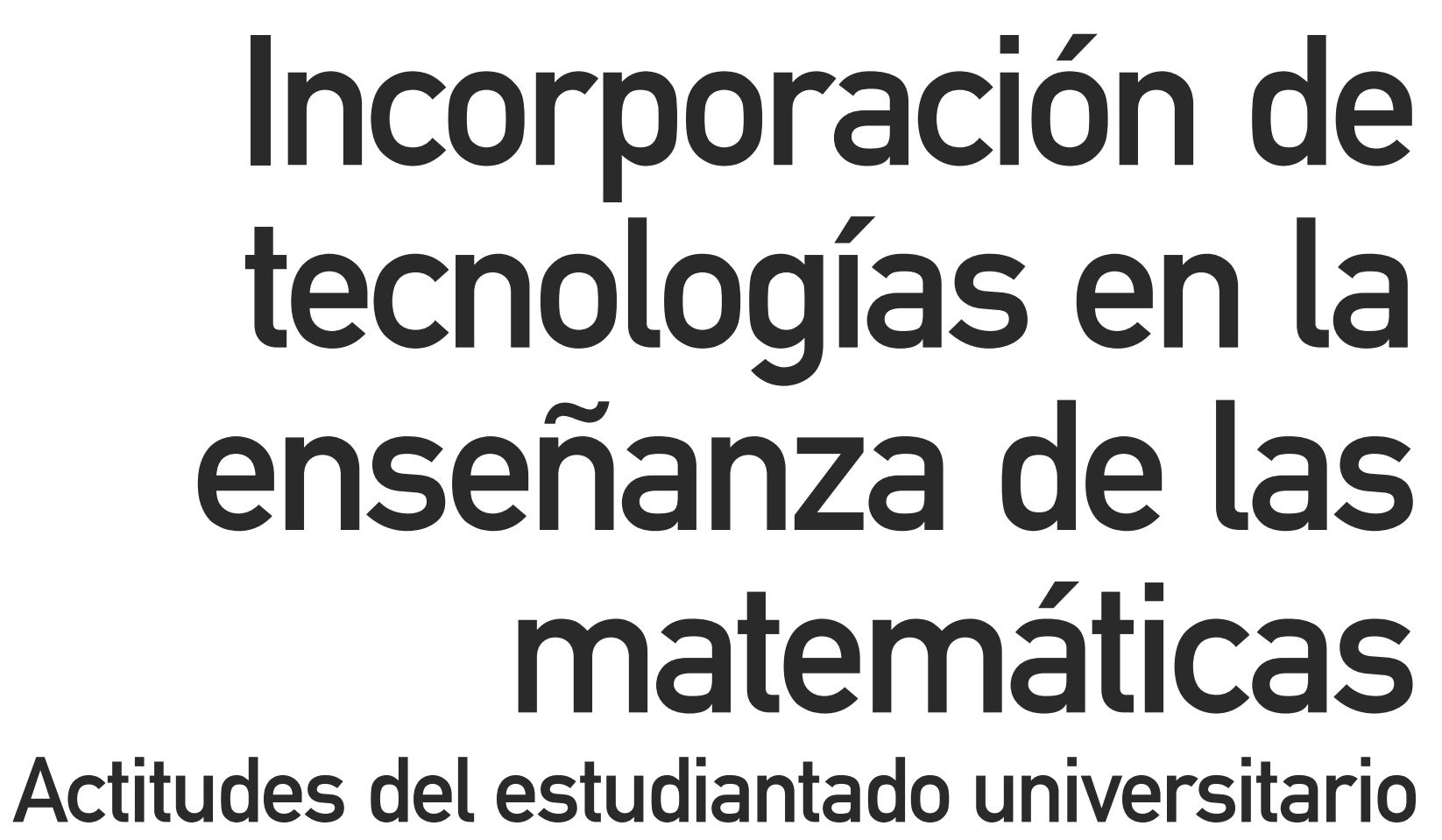

Incorporating technologies in math teaching: Attitudes of higher education students

William Oswaldo Flores López

Una de las competencias que debe tener el ciudadano de la sociedad del conocimiento, es la competencia tecnológica, ya que los sujetos que no sepan desenvolverse con la tecnología digital de un modo inteligente (saber conectarse y navegar por redes, buscar la información útil, analizarla y reconstruirla, comunicarla a otros usuarios) no podrán acceder a la cultura y al mercado de la sociedad de la información (Area Moreira, Gros Salvat, \& Marzal García-Quismondo, 2008, pág. 39). Por ello, las actitudes del estudiantado ante el proceso de enseñanza y aprendizaje de las matemáticas con tecnología es un tema que ha despertado el interés de investigadores como Galbraith y Haines (2000), Nguyen y Kulm (2005), Cretchley (2007), Pierce, Stacey y Barkatsas (2007) y López, Castro Martínez y Molina (2013).

En este sentido, el objetivo central de esta investigación ha sido analizar las actitudes del estudiantado ante la incorporación de tecnologías en la enseñanza de las matemáticas en los grados en ingeniería civil, informática administrativa y matemáticas. Se ha formulado este objetivo fijando la atención en la incorporación de las tecnologías de la comunicación e información en la enseñanza universitaria. Lo que conlleva a formular los objetivos específicos siguientes: explicar si existe relación entre las categorías hacia el uso de la tecnología en el aprendizaje de las matemáticas; estudiar si existen diferencias estadísticamente significativas entre género y edad en relación con las categorías de actitudes hacia el uso de la tecnología en el aprendizaje de las matemáticas; e identificar si existen diferencias estadísticamente significativas en la variable especialidad en relación con las actitudes globales hacia la incorporación de tecnologías en la enseñanza de las matemáticas. 


\section{Actitudes hacia las matemáticas y tecnologías}

La evaluación de las actitudes del estudiantado hacia una disciplina, un contenido específico, una metodología o cualquier herramienta didáctica, es un tema de interés tanto para la investigación científica, como para la práctica educativa. En la educación y su campo de acción, cada vez con más frecuencia, se habla de la notable influencia que ejercen las variables afectivas sobre el rendimiento académico del estudiantado universitario. Varios investigadores analizan la relación entre estados afectivos y cognitivos desde la perspectiva de la influencia en la resolución de problemas y rendimiento académico. (Auzmendi Escribano, 1992; Gil, Gil Blanco, \& Guerrero, 2005; Gómez Chacón, 2009; Martínez Padrón, 2008; Petriz Mayen, Barona Ríos, López Villareal, \& Quiroz González, 2010; Alvarez \& Ruíz Soler, 2010; Pérez Tyteca, Castro Martínez, Rico Romero, \& Castro Martínez, 2011; Cardoso Espinosa, Vanegas López, \& Cerecedo Mercado, 2012; Flores López \& Auzmendi Escribano, 2015). Las variables afectivas constituyen una vasta categoría de sentimientos y estados de ánimo que incluyen elementos como las actitudes, las creencias y las emociones. (McLeod \&Adams, 1989)

En relación con las actitudes, que es el foco de interés de esta investigación, existe un consenso entre los teóricos en definirlas como una predisposición con cierta carga emocional, que influye en la conducta del ser humano, esta definición remarca tres componentes básicos de la actitud: cognición o creencias sobre el objeto actitud, el afecto o carga evaluativa de dichas creencias y una intención de conducta o relación a dicha actitud (Gil, Gil Blanco, \& Guerrero, 2005). En referencia con las matemáticas, cabe distinguir entre actitudes matemáticas y actitudes hacia las matemáticas. La actitud hacia las matemáticas tendría quevercon lavaloración, el aprecio, la satisfacción, la curiosidad y el interés tanto por la disciplina como por su aprendizaje, acentuando más el componente afectivo que el cognitivo (Martínez Padrón, 2008). Las actitudes matemáticas, por el contrario, tendría que ver con el modo de utilizar capacidades generales como la reflexión de pensamiento, apertura mental, el espíritu crítico, la objetividad, etc., que son importantes en el trabajo en matemáticas. (Gómez Chacón, 2009)

En Petriz, Barona Ríos, López Villareal y Quiroz González (2010), se define específicamente la actitud hacia las matemáticas como una serie de disposiciones que manifiesta el individuo para aceptar familiarizarse, o no, con determinados contenidos matemáticos. En su investigación describieron que los estudiantes con mayor motivación hacia las matemáticas alcanzaron un mayor nivel de desempeño. Concluyeron, además, que una dosis moderada de ansiedad origina desempeños de alto rendimiento. Además, concluyeron que la preparación de los docentes para esta asignatura se concentra más en los aspectos de contenido, en detrimento de las actitudes, las cuales son necesarias aprender a diagnosticar para, así, contar con la posibilidad de implementar diseños instruccionales apropiados para una educación matemática de calidad.

Por su parte, Pérez Tyteca, Castro Martínez, Rico Romero y Castro Martínez (2011), conceptualizan la actitud hacia las matemáticas como la predisposición aprendida por el estudiantado para responder de manera positiva o negativa ante las matemáticas, lo que determina su intención e influye en su comportamiento ante la materia. Así, en su estudio, concluyeron que existen diferencias significativas entre hombres y mujeres en lo que respecta a su ansiedad ante las matemáticas, en donde los primeros son los que sufren menos ansiedad al enfrentarse a las actividades matemáticas. Por su parte,
Álvarezy Ruiz Soler (2010), encontraron que los estudiantes de ingeniería manifiestan una actitud globalmente positiva, o sea, reconocen la importancia de las matemáticas en su formación académica y sienten curiosidad e interés por resolver problemas relacionados con los estudios que están realizando. También señalan no sentir entusiasmo, emoción o pasión por las matemáticas. No obstante, admiten que algunos estudiantes presentan dificultad con la disciplina en la medida que no la comprenden, sienten incomodidad, nerviosismo y se sienten incapaces de pensar con claridad ante este tipo de situaciones.

En analogía con la actitud hacia las tecnologías puede ser entendida como una disposición aprendida, más o menos permanente, la cual se expresa en términos de las creencias, los sentimientos y la tendencia a la acción del estudiantado hacia estas tecnologías en sentido favorable, neutro o desfavorable (Bolívar Ruiz, 2010). La actitud favorable está integrada por aquellos estudiantes que están muy entusiasmado sobre el tema y están involucrados de manera activa con el mismo. La actitud neutra está representada por aquellos estudiantes que están medianamente interesados y pudieran ser entusiasmados bajo incentivos y circunstancias apropiadas. La actitud desfavorable está formada por el estudiantado que siente que las tecnologías no tienen una alta prioridad y que no vale la pena hacer esfuerzo al respecto. Desde esta perspectiva, investigadores como Hoyles y Sutherland (1989), Balacheff y Kaput (1996), Dettori, Garuti y Lemut (2001), Mariotti (2005), Ursini, Sánchez y Orendain (2004) y, Flores y López (2016), han explorado posibilidades para mejorar la enseñanza y el aprendizaje de las matemáticas en los distintos niveles educativos mediante el uso de tecnología, reportando que, al trabajar temas de matemáticas incorporando tecnologías, aumenta significativamente la motivación del estudiantado hacia el aprendizaje de las matemáticas, registrando un cambio positivo en las actitudes hacia esta materia.

En cambio, Vale (2001) y, Volman y Van Eck (2001), investigan el comportamiento y las actitudes del estudiantado hacia las computadoras al hacer uso de ellos en clases de matemáticas; Pierce (2002), analiza las actitudes del estudiantado hacia el uso de software en su aprendizaje y los distintos usos que los estudiantes hacen de él; y Depool Rivero (2005), observa que el uso del software denominado Derive en actividades de cálculo integral es valorado como útil e interesante por el estudiantado; los resultados de dichas investigaciones señalan que la integración de las tecnologías en la enseñanza y aprendizaje de las matemáticas mejora el aprendizaje, aumenta la participación del estudiantado, y favorece el desarrollo de la motivación intrínseca.

Para finalizar, señalar que en los últimos años los instrumentos de evaluación de las actitudes están proliferando en escalas de medidas para identificar las actitudes y la ansiedad hacia las matemáticas y estadísticas. Dichas escalas tratan de medir dimensiones o factores actitudinales como ansiedad, agrado, motivación, utilidad y confianza hacia las matemáticas (Flores López \& Auzmendi Escribano, 2015). En este sentido, se ha elegido el cuestionario de actitudes hacia las matemáticas y tecnología de López, Castro Martínez y Molina (2013), porque mide: la interacción ordenador y matemáticas; las actitudes hacia el aprendizaje de las matemáticas con tecnología; las actitudes del uso de las tecnologías en el aprendizaje de las matemáticas; las experiencias matemáticas con tecnología; y, la matemática y tecnología.

\section{Metodología}

El enfoque de esta investigación es cuantitativo porque este paradigma ayudó al tratamiento de la información de los datos a través de la categorización y descripción de las propiedades, características y los perfiles de las personas, grupos, comunidades, procesos y objetos o 
cualquier otro fenómeno que se someta a un análisis (Hernández Sampieri, Fernández Collado, \& Baptista Lucio, 2010). Mientras que el diseño que se instauro es un diseño descriptivo ya que tiene como objetivo indagar la incidencia de las modalidades o niveles de una o más población. (Bisquerra Alzina, 2009)

El estudio se llevó a cabo con muestra de 106 estudiantes universitarios de los grados en Ingeniería Civil, Informática y Matemáticas de la Universidad de las Regiones Autónomas de la Costa Caribe Nicaragüense, pertenecientes a comunidades de la Costa Caribe de Nicaragua. Para concluir indicar que el tipo de muestreo que hemos utilizado ha sido el muestro no probabilístico causal o accidental, que es aquel en el cual el investigador selecciona directa e intencionalmente la muestra, debido fundamentalmente a que tiene fácil acceso a la misma y es representativa de la población. (Gil Flóres, Rodríguez Gómez, \& García Jiménez, 1995; Albert Gómez, 2007; Sabariego Puig, 2004)

El instrumento para la recopilación de la información es el cuestionario de actitudes hacia el uso de la tecnología en el aprendizaje de las matemáticas de López, Castro Martínez y Molina (2013). El instrumento está constituido por 35 ítems, aglutinados en 5 dimensiones. El estudiantado debía indicar su grado de acuerdo o desacuerdo con afirmaciones propuestas por medio de una escala de Likert de 5 valores. A continuación, se describen las 5 dimensiones 0 categorías del instrumento:

Categoría 1. Interacción ordenadory matemáticas. Constituida por 8 ítems que involucran la utilidad del uso de la tecnología en hacer y aprender matemáticas, y creencias del estudiantado acerca de su aprendizaje de las matemáticas con tecnología. (Galbraith \& Haines, 2000)

Categoría 2. Actitudes hacia el aprendizaje de las matemáticas con tecnología. Integrada por 4 ítems que miden el gusto y la motivación del estudiantado hacia las matemáticas. (Pierce, Stacey, \& Barkatsasb, 2007)

Categoría 3. Actitudes del uso de las tecnologías en el aprendizaje de las matemáticas. Este tercer bloque está integrado por 12 ítems que miden los sentimientos de gusto y motivación al uso de tecnología cuando se hace y se aprende matemáticas. (Cretchley, 2007)

Categoría 4. Experiencias de matemáticas con tecnología. Establecida por 5 ítems que evalúan las actitudes del estudiantado referente con su experiencia personal del uso de software en su aprendizaje de las matemáticas. (Cretchley, 2007)

Categoría 5. Matemática y tecnología. Integrada por 6 ítems que miden el gusto por la integración de la tecnología en las matemáticas. (Nguyen \& Kulm, 2005)

Para garantizar la calidad de la medida, se aplicó un estudio socio métrico al instrumento, para comprobar los valores de validez y fiabilidad del mismo. Se calculó el valor de consistencia interna del total de los ítems del cuestionario mediante alfa de Cronbach, obteniendo el valor de $\mathbf{9 0 \%}$ de confiabilidad. Con respecto a la validez, se realizó un análisis de componentes principales (ACP). La prueba Kaiser-MeyerOlkin arrojo una puntuación de $\mathbf{0 . 8 0 1}$. Por su parte, la prueba de esfericidad también ofreció resultados que indicaban que el análisis era pertinente (Chi-cuadrado: $X^{2}=1970.219 ;$ g.l. $=595 ; p<0.000$ ). Además, se calculó el determinante de la matriz de correlaciones, cuyo valor fue prácticamente $0(D=5.619 E-10)$.

La administración del instrumento se realizó por parte del autor durante el curso académico 2016. Tenía un carácter anónimo y fue complementado por los sujetos participantes en presencia del profesorado. Con anterioridad a la toma de los datos, se obtuvo tanto el consentimiento libre e informado del estudiantado, profesorado, así como la autorización de las autoridades universitarias.
Para satisfacer los objetivos del estudio se procedió al desarrollo de distintos análisis empleando el paquete estadístico para las ciencias sociales SPSS. Entre ellos se aplicó el análisis de la consistencia interna del cuestionario del cuestionario, el análisis estructural del cuestionario, un estudio descriptivo de los datos, así como un estudio de correlaciones entre las categorías, prueba T-Student y análisis de varianza con prueba HSD-Tukey.

\section{Resultados}

El $\mathbf{3 0} \%$ (32) de las participantes son mujeres, frente al $\mathbf{7 0} \%$ (74) que son hombres. Respectoasuedad, lamediasesitúaen21años,encontrándose el $\mathbf{8 0 . 8 6 \%}$ de los participantes en el intervalo de edad entre 18-22 años. En referencia a la especialidad que cursa el estudiantado en la universidad, el $\mathbf{2 7} \%$ estudian el grado en Matemáticas, el $\mathbf{2 1} \%$ cursan la especialidad en Informática, frente al $\mathbf{5 2} \%$ restante que desarrollan sus estudios en la especialidad en Ingeniería Civil (Véase la Gráfico 1). Otras características de los participantes son: la gran mayoría de los participantes (97\%) encuestados poseen la competencia digital; el $\mathbf{9 3}$ \% tienen en propiedad un dispositivo móvil (Smartphone, Tablet...); $\mathbf{9 0 \%}$ disponen de internet, conectándose habitualmente desde la universidad o en cualquier otro lugar ya disponen de internet móvil un 10\%; y el tiempo que dedican a la conexión es de más 10 horas semanales para el $\mathbf{7 5} \mathbf{\%}$ de los participantes, frente a un $\mathbf{2 0} \mathbf{\%}$ que se conecta entre 5-10 horas semanales y sólo el $\mathbf{5} \%$ de la muestra se conecta a internet una hora o menos por semana.

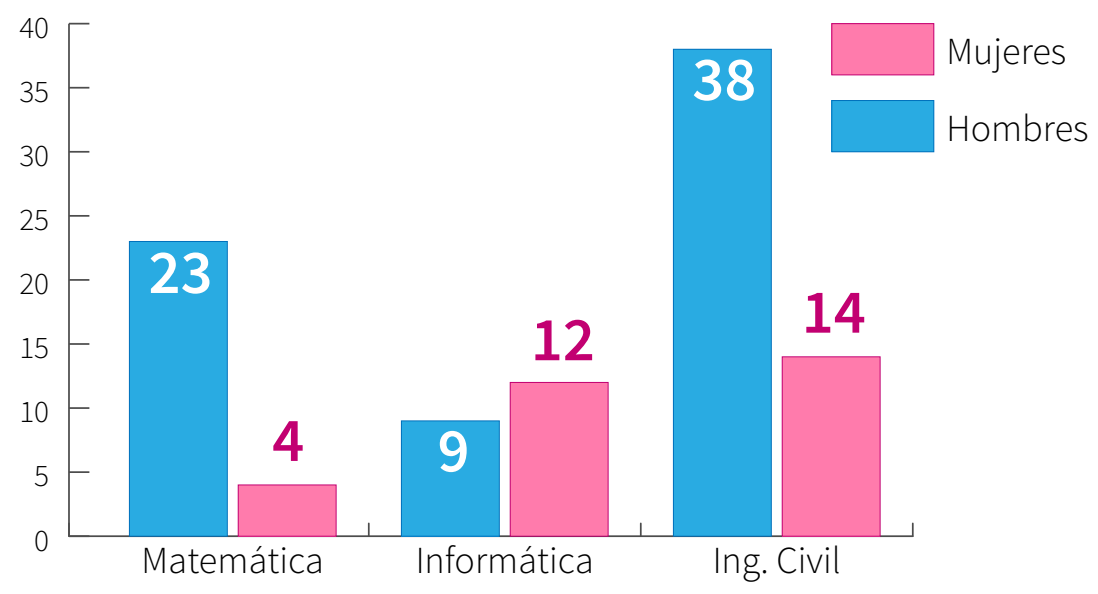

Gráfico 1 Distribución de los participantes en función de la variable especialidad

\section{Actitudes del estudiantado universitario hacia la incorporación de tecnologías en la enseñanza y aprendizaje de las matemáticas}

A partir de las distintas categorías del cuestionario, se calculó la puntuación que obtendría cada participante de la muestra en cada una de las escalas. El resultado se analiza desde un análisis descriptivo, indicando los niveles de actitud del estudiantado participante en relación con los temas de interés de esta investigación. Primeramente, se analizó la puntuación total de la escala, así como las distintas agrupaciones, según las categorías que se asocian en el cuestionario de actitudes hacia el uso de la tecnología en el aprendizaje de las matemáticas. 
Los resultados muestran que la puntuación total del cuestionario es media con $\mathbf{1 1 7 . 0 8}$ puntos ( $S D=18.53$; Error $=1.8$. . A la vista de este resultado podemos afirmar que las actitudes hacia el uso de la tecnología en el aprendizaje de las matemáticas son positiva globalmente. Así como, al no alcanzarse valores extremos en la puntuación, la gran mayoría se concentra en una actitud positiva, alrededor de $\mathbf{1 2 9}$ (moda), actitud que podríamos considerar moderada con tendencia alta.

\section{Tabla 1 Interacción ordenador y matemáticas}

\section{No} Ítems

Las computadoras me ayudan a aprender mejor las matemáticas

1 proporcionándome al instante muchos ejemplos de manera

$3.57 \quad 1.16$ interactiva.

Me resulta difícil comprender la transferencia de ideas de la pantalla de una computadora a mi mente.

El no tener que preocuparme por los cálculos aritméticos,

3 hace que me concentre mejor en las ideas esenciales de las matemáticas.

Cuando leo la pantalla de una computadora, tengo la tendencia a pasar por alto detalles de las ideas matemáticas. Considero que el material impreso en la pantalla de una

5 computadora y la copia impresa en el papel es útil para tomar $\quad \begin{array}{ll}3.92 & 0.92\end{array}$ notas.

6 Rara vez reviso el material inmediatamente después de que una $\quad 3.10 \quad \begin{array}{ll}1.12\end{array}$ sesión por computadora ha terminado.

El seguimiento de las instrucciones tecleadas pone atención fuera de las matemáticas.

Las computadoras me ayudan a vincular el conocimiento, como, por ejemplo, la forma de los gráficos y sus ecuaciones

$4.16 \quad 0.99$

Fuente: elaboración propia

Sobre un máximo de $\mathbf{4 0}$ puntos, la muestra presenta una media de $\mathbf{2 6 . 3 3}$ puntos obtenidos en la categoría interacción ordenador y matemáticas $(S D=4.08$; Error $=0.39)$. El $\mathbf{5 0} \%$ de los participantes se situó por debajo de los $\mathbf{3 2}$ puntos, con una moda de $\mathbf{2 7}$. Estos datos indican que la muestra tiende a presentar una distribución de puntuaciones medias altas. El coeficiente de variación obtenido en la categoría fue del $\mathbf{1 6 . 6 8}$. En esta categoría las puntuaciones de los ítems oscilan entre $\mathbf{2 . 5 0}$ a $\mathbf{4 . 1 6}$. La puntuación más alta fue alcanzada en el ítem 8 (Las computadoras me ayudan a vincular el conocimiento, como, por ejemplo, la forma de los gráficos y sus ecuaciones) con una media de $\mathbf{4 . 1 6}$ de las réplicas emitidas. Por el contrario, el ítem 2 (Me resulta difícil comprender la transferencia de ideas de la pantalla de una computadora a mi mente) con $\mathbf{2 . 5 0}$, así como el ítem 3 (El no tener que preocuparme por los cálculos aritméticos, hace que me concentre mejor en las ideas esenciales de las matemáticas).

Tabla 2 Actitudes hacia el aprendizaje de las matemáticas con tecnología

\begin{tabular}{|cccc}
\hline No & Ítems & & \\
\hline 9 & Me gusta usar computadoras en matemáticas & 3.46 & 1.35 \\
10 & $\begin{array}{r}\text { Vale la pena el esfuerzo adicional del uso de computadoras en } \\
\text { matemáticas. }\end{array}$ & 3.81 & 1.13 \\
11 & Matemáticas es más interesante cuando usas computadoras & 3.36 & 1.38 \\
12 & Las computadoras me ayudan a aprender mejor las matemáticas & 3.57 & 1.18
\end{tabular}

Fuente: elaboración propia

Respecto con la categoría actitudes hacia el aprendizaje de las matemáticas con tecnología la puntuación media de los participantes fue de $\mathbf{1 4 . 2 2}$ puntos $(S D=4.16$; Error=.4) sobre un máximo de $\mathbf{2 0}$ puntos, situándose en el rango en los $\mathbf{1 6}$ puntos. Con una varianza de $\mathbf{1 7 . 3 7 \%}$. La tendencia de las puntuaciones de los ítems oscila entre $\mathbf{3 . 3 6}$ el ítem 11 (Matemáticas es más interesante cuando usas computadoras) y $\mathbf{3 . 8 1}$ el ítem 10 (Vale la pena el esfuerzo adicional del uso de computadoras en matemáticas). Se concluye, que el estudiantado posee una actitud media alta en referencia con las actitudes hacia el aprendizaje de las matemáticas con tecnología.

Tabla 3 Actitudes del uso de la tecnología en el aprendizaje de las matemáticas

No

13 El poder de la computación hace más fácil explorar ideas matemáticas

14 Las computadoras están cambiando el modo de hacer matemáticas

Sé que las computadoras son importantes, pero no siento la necesidad de usarlas para aprender matemáticas

6 Las computadoras son buenas herramientas para los cálculos, pero no para mi aprendizaje de matemáticas

17 Pienso que el uso de las tecnologías es demasiada nuevo y extraño para que valga la pena en el aprendizaje

Pienso que el uso de la tecnología es una pérdida de tiempo en el 18 aprendizaje de las matemáticas

19 Prefiero hacer todos los cálculos y gráficas manualmente sin tener que usar una computadora

20 El uso de la tecnología para cálculos me facilita hacer las aplicaciones más realísticas

Me gusta explorar métodos matemáticos e ideas usando tecnología

2 Disponer de la tecnología para hacer el trabajo rutinario me 22 permite probar diferentes métodos y enfoques.

23 Quiero mejorar en el uso de las computadoras para que me ayude en las matemáticas complicados sin la adición de la tecnología

Con un máximo de $\mathbf{6 0}$ puntos se presenta la categoría actitudes del uso de la tecnología en el aprendizaje de las matemáticas, la media de la muestra se situó en $\mathbf{3 8 . 9 5}$ puntos con una mediana de $\mathbf{3 9}$ puntos. El errortípico de la media fue de $\mathbf{0 . 6 1}$, junto con una desviación estándar de $\mathbf{6 . 3 1}$. La moda fue de $\mathbf{4 0}$ puntos coincidiendo con la mediana. Por tanto, la distribución se presentó algo más simétrica que las categorías anteriores con un signo negativo similar. El coeficiente de variación obtenido en la categoría fue del $\mathbf{3 9 . 8 9} \%$. Destacar en esta categoría, que los valores más seleccionados por parte del estudiantado son: el ítem 22 con 3.91 (Disponer de la tecnología para hacer el trabajo rutinario me permite probar diferentes métodos y enfoques.) y ítem 23 con $\mathbf{3 . 9 1}$ (Quiero mejorar en el uso de las computadoras para que me ayude en las matemáticas). Para un contraste del ítem 18 con $\mathbf{2 . 2 1}$ (Pienso que el uso de la tecnología es una pérdida de tiempo en el aprendizaje de las matemáticas) y el ítem 17 con $\mathbf{2 . 6 1}$ (Pienso que el uso de las tecnologías es demasiada nuevo y extraño para que valga la pena en el aprendizaje). En resumen, el estudiantado universitario posee una actitud positiva (media alta) hacia el uso de las tecnologías en el aprendizaje de las matemáticas.

\section{Tabla 4 Experiencias matemáticas con tecnologías}

\begin{tabular}{|c|c|c|c|}
\hline$N^{0}$ & Ítems & & \\
\hline 25 & $\begin{array}{l}\text { He encontrado software útil para mi aprendizaje de las } \\
\text { matemáticas }\end{array}$ & 3.34 & 1.20 \\
\hline 26 & Aprender a usar software para hacer matemáticas es frustrante & 2.95 & 1.22 \\
\hline 27 & $\begin{array}{l}\text { El uso de software hace el aprendizaje de las matemáticas más } \\
\text { interesantes }\end{array}$ & 3.50 & 1.18 \\
\hline 28 & $\begin{array}{l}\text { En términos generales vale la pena aprender a utilizar el software } \\
\qquad \text { para hacer matemáticas }\end{array}$ & 3.62 & 1.15 \\
\hline 29 & $\begin{array}{l}\text { Por propia elección usaré las veces que sea necesario software } \\
\qquad \text { para matemáticas }\end{array}$ & 3.50 & 1.19 \\
\hline
\end{tabular}

Fuente: elaboración propia

En la categoría experiencias matemáticas con tecnologías, los participantes obtuvieron una distribución con media de $\mathbf{1 6 . 9 3}$ (SD = 4.17) y un error estándar de $\mathbf{0 . 4 0}$. El rango fue de $\mathbf{2 0}$ con un máximo de $\mathbf{2 5}$ y un mínimo de $\mathbf{5}$. El rango intercuartílico fue de $\mathbf{1 7}$ puntos, situándose la mediana en los $\mathbf{1 7}$ puntos. De esta forma, la distribución de frecuencias presento una simetría negativa y una curtosis positiva, 
siendo el coeficiente de variación de $\mathbf{1 7 . 4 3} \%$. En general, se aprecia que el estudiantado tiene una actitud media alta con las experiencias matemáticas con tecnología.

\section{Tabla 5 Matemáticas y tecnología}

\begin{tabular}{|c|c|c|c|}
\hline & Ítems & & \\
\hline 30 & Me gusta aprender matemáticas con la ayuda de la computadora & 3.60 & 1.18 \\
\hline 31 & $\begin{array}{l}\text { Las tareas matemáticas por computadora son claras y fáciles de } \\
\text { leer }\end{array}$ & 3.51 & 1.18 \\
\hline 32 & $\begin{array}{l}\text { La retroalimentación inmediata de la computadora es útil para } \\
\text { resolver problemas matemáticas }\end{array}$ & 3.52 & 1.1 \\
\hline 33 & $\begin{array}{l}\text { La revisión de la lección en la tarea por computadora me ayuda a } \\
\text { repasar los conceptos matemáticos }\end{array}$ & 3.44 & 1.18 \\
\hline 34 & $\begin{array}{l}\text { Los exámenes de matemáticas por computadora con puntuación } \\
\text { inmediata me ayudan a evaluar mi propio entendimiento y } \\
\text { rendimiento. }\end{array}$ & 3.44 & 1.28 \\
\hline 35 & $\begin{array}{l}\text { Me gustan las pruebas de matemáticas por computadoras más } \\
\text { que las pruebas de lápiz y papel. }\end{array}$ & 3.10 & 1.44 \\
\hline
\end{tabular}

Fuente: elaboración propia

Con una puntuación máxima de $\mathbf{3 0}$ puntos, se presenta la categoría 5. Matemática y tecnología; la media de la muestra se situó en los $\mathbf{2 0 . 6 4}$ puntos con una mediana de $\mathbf{2 1}$ puntos. El error típico de la media fue de $\mathbf{0 . 5 7 3}$ junto con una desviación estándar $\mathbf{5 . 8 9}$. El coeficiente de variación obtenido en la categoría fue del $\mathbf{3 4 . 8 0} \%$. Destacar que los ítems están en rango de $\mathbf{2 4}$, con una oscilación entre $\mathbf{3 . 1 0}$ (Me gustan las pruebas de matemáticas por computadoras más que las pruebas de lápiz y papel) y $\mathbf{3 . 6 0}$ (Me gusta aprender matemáticas con la ayuda de la computadora). En definitiva, el estudiantado tiene una actitud media alta hacia la matemáticas y tecnologías.

\section{Relaciones entre las categorías de actitud hacia el uso de tecnologías en la enseñanza y aprendizajes de las matemáticas en la universidad}

Se consideró a las categorías como una variable, para estudiar las correlaciones entre ellas (Tabla 6), se obtuvieron coeficientes de correlación en una oscilación entre $\mathbf{0 . 2 9 4}$ y $\mathbf{0 . 6 7 4}$. La categoría 1 , ordenador y matemáticas se asocia con actitudes hacia el aprendizaje de las matemáticas con tecnología (Categoría 2) con $\mathbf{0 . 4 4 6}$, también la categoría $\mathbf{1}$, ordenador y matemáticas se asocia con las actitudes del uso de las tecnologías en el aprendizaje de las matemáticas (Categoría 3) con $\mathbf{0 . 3 2 6}$. Igualmente, interacción ordenador y matemáticas se correlaciona con las experiencias matemáticas con tecnología (Categoría 4) con $\mathbf{0 . 4 2 9}$. Sin embargo, interacción ordenador y matemáticas se relaciona con matemática y tecnología (Categoría 5) con una correlación baja de $\mathbf{2 . 9 4}$.

En la categoría 2, actitudes hacia el aprendizaje de las matemáticas con tecnología se asocian con las actitudes del uso de las tecnologías en el aprendizaje de las matemáticas (Categoría 3) con $\mathbf{0 . 3 0 2}$, así también con experiencias matemáticas con tecnología (Categoría 4) con $\mathbf{0 . 6 5 4}$; y con la categoría 5 , matemáticas y tecnología con una correlación de $\mathbf{0 . 6 5 2}$. En la categoría 3, existe una asociación de $\mathbf{0 . 4 8 3}$, entre las actitudes del uso de las tecnologías en el aprendizaje de las matemáticas (Categoría 3) y experiencias matemáticas con tecnología (Categoría 4); además las actitudes del uso de las tecnologías en el aprendizaje de las matemáticas (Categoría 3) se relaciona con matemáticas y tecnología (Categoría 5) con $\mathbf{0 . 3 6 3}$. Así como, se dan correlaciones entre experiencias matemáticas con tecnología (Categoría 4) y, matemática y tecnología (Categoría 5) con $\mathbf{0 . 6 7 4 .}$

\begin{tabular}{|cccccc|} 
Tabla 6 Relaciones entre las categorías del cuestionario de actitud \\
\hline Categorias & $\mathbf{1}$ & $\mathbf{2}$ & $\mathbf{3}$ & $\mathbf{4}$ & $\mathbf{5}$ \\
\hline 1 & 1 & 0.446 & 0.326 & 0.429 & 0.294 \\
2 & & 1 & 0.302 & 0.654 & 0.652 \\
3 & & & 1 & 0.483 & 0.363 \\
4 & & & 1 & 0.674 \\
5 & & & & 1 \\
& & & & Fuente: elaboración propia \\
\hline
\end{tabular}

\section{Género, edad, y especialidad son determinantes en la enseñanza y aprendizaje de las matemáticas con tecnologías en la universidad}

Con el objetivo de reforzar al lector la visión de los resultados obtenidos, se presenta en la Tabla 7, las puntuaciones promedias alcanzadas por cada categoría en función de la variable género, indicando los valores promedios alcanzados en la puntuación media de las categorías.

Tabla 7 Medias de las categorías en función de la variable género

\begin{tabular}{|ccc|}
\hline Categorías & \multicolumn{2}{c|}{ Media } \\
\hline $\begin{array}{c}\text { Interacciones ordenador y matemáticas } \\
\text { Actitudes hacia el aprendizaje de las matemáticas con } \\
\text { tecnología }\end{array}$ & 26.68 & 25.53 \\
$\begin{array}{c}\text { Actitudes del uso de las tecnologías en el aprendizaje de las } \\
\text { matemáticas }\end{array}$ & 14.49 & 13.59 \\
\hline $\begin{array}{c}\text { Experiencias de matemáticas con tecnología } \\
\text { Matemática y tecnología }\end{array}$ & 17.11 & 39.55 \\
& 20.58 & 16.53 \\
& Fuente: elaboración propia \\
\hline
\end{tabular}

Se realizó un análisis de puntuaciones medias de las categorías en función de la variable género. La prueba T-Student para muestras independientes confirman que en las categorías: interacción ordenador y matemáticas ( $p=0.187$ ); actitudes hacia el aprendizaje de las matemáticas con tecnología ( $p=0.314$ ); actitudes del uso de la tecnología en el aprendizaje de las matemáticas ( $p=0.325)$; experiencias matemáticas con tecnologías ( $p=0.516)$; y matemática y tecnología ( $p=0.873$ ), hombres y mujeres poseen las mismas actitudes hacia el proceso de enseñanza y aprendizaje de las matemáticas con tecnologías, ya que los valores de significancia p es mayor que 0.05.

\begin{tabular}{|ccc|}
$\begin{array}{c}\text { Tabla } 8 \text { Medias de las categorías en función de la variable edad } \\
\text { Categorías }\end{array}$ & \multicolumn{2}{c|}{ Media } \\
\hline $\begin{array}{c}\text { Interacciones ordenador y matemáticas } \\
\text { Actitudes hacia el aprendizaje de las matemáticas con }\end{array}$ & 25.59 & 26.80 \\
$\begin{array}{c}\text { tecnología } \\
\text { Actitudes del uso de las tecnologías en el aprendizaje de las } \\
\text { matemáticas }\end{array}$ & 13.23 & 14.98 \\
\hline $\begin{array}{c}\text { Experiencias de matemáticas con tecnología } \\
\text { Matemática y tecnología }\end{array}$ & 15.93 & 39.69 \\
\hline & 18.39 & 22.31 \\
\hline
\end{tabular}

Fuente: elaboración propia

La Tabla 8, muestra las puntuaciones medias de las categorías en función de la variable edad, se puede observar que los estudiantes entre las edades 19 y 20 poseen una mayor actitud hacia las matemáticas con incorporación de tecnologías en la enseñanza y aprendizaje de dicha asignatura. Esto se confirma con la prueba de T-Student en las categorías: actitudes hacia el aprendizaje de las matemáticas con tecnología ( $p=0.033$ ); experiencias matemáticas con tecnologías ( $p=0.041)$; $y$, matemática y tecnología $(p=0.001)$, es decir, existen diferencias estadísticamente significativas entre las edades 17-18 y 1920 a favor del estudiantado con edades 19-20, porque los valores de 


\section{Incorporación de tecnologías en la enseñanza de las matemáticas}

\section{Actitudes del estudiantado universitario}

significancia $p$ es menor que 0.05 . Por otra parte, los resultados de la prueba T-Student también muestra que en la interacción ordenador y matemáticas ( $p=0.134)$; y actitudes del uso de la tecnología en el aprendizaje de las matemáticas ( $p=0.163$ ), que las edades entre 17 a 20 tienen el mismo grado actitudes hacia el aprendizaje de las matemáticas con tecnologías, ya que los valores de significancia $p$ es mayor que 0.05 .

También, se trató de estudiar si existen diferencias significativas entre especialidad en relación con las actitudes hacia el uso de la tecnología en el aprendizaje de las matemáticas (puntuación total del cuestionario), el análisis de varianza de un factor se encontró que ( $p=0.062)$, teniendo en cuenta que $(p>0.05)$, se puede decir que no existen diferencias estadísticamente significativas, concluyendo que las especialidades en ingeniería civil, informática y matemática tienen el mismo grado de actitud hacia el uso de la tecnología en el aprendizaje de las matemáticas.

\section{Tabla 9 Comparaciones medias Post hoc HSD-Tukey}

\begin{tabular}{|c|c|c|c|c|c|c|}
\hline \multirow{2}{*}{$\begin{array}{c}\text { (I) } \\
\text { Especialidad }\end{array}$} & \multirow{2}{*}{$\begin{array}{c}(\mathrm{J}) \\
\text { Especialidad }\end{array}$} & \multirow{2}{*}{$\begin{array}{c}\text { Diferencia de } \\
\text { medias (I-J) }\end{array}$} & \multirow{2}{*}{$\begin{array}{l}\text { Error } \\
\text { estándar }\end{array}$} & \multirow[t]{2}{*}{ Sig. } & \multicolumn{2}{|c|}{$\begin{array}{l}95 \% \text { intervalo } \\
\text { de confianza }\end{array}$} \\
\hline & & & & & L-inf. & L-sup. \\
\hline \multirow{2}{*}{ Matemática } & Informática & -11.008 & 5.125 & 0.085 & -23.20 & 1.18 \\
\hline & Ingeniería Civil & -1.255 & 4.228 & 0.953 & -11.31 & 8.80 \\
\hline \multirow{2}{*}{ Informática } & Matemática & 11.008 & 5.125 & 0.085 & -1.18 & 23.20 \\
\hline & Ingeniería Civil & 9.753 & 4.522 & 0.084 & -1.00 & 20.51 \\
\hline \multirow{2}{*}{$\begin{array}{l}\text { Ingeniería } \\
\text { Civil }\end{array}$} & Matemática & 1.255 & 4.228 & 0.953 & -8.80 & 11.31 \\
\hline & Informática & -9.753 & 4.522 & 0.084 & -20.51 & 1.00 \\
\hline
\end{tabular}

Fuente: elaboración propia

No obstante, la prueba post hoc (HSD-Tukey) manifiesta que el estudiantado del grado en informática posee una mayor actitud hacia el uso de la tecnología en el aprendizaje de las matemáticas en comparación con el estudiantado de ingeniería civil y matemáticas (véase Tabla 7).

\section{Discusión}

La actitud del estudiantado globalmente es moderada con tendencia alta $(M=117.08 ; S D=18.53 ;$ Error=1.8), concordando con los estudios de Sánchez Ruiz y Ursini (2010) realizado con estudiantes mexicanos donde encontraron que la actitud del estudiantado ante la incorporación de tecnología en la enseñanza de las matemáticas es media con tendencia neutral, es decir, la mayoría del estudiantado manifiesta una actitud positiva hacia el uso de la tecnología. Así mismo, concertando con los estudios de Cretchley (2007), Pierce, Stacey y Barkatsas (2007) y, Gómez Chacón y Haines (2008) que reportaron que, al trabajar temas de matemáticas con el apoyo de la tecnología, aumenta notablemente la motivación del estudiantado hacia el aprendizaje de las matemáticas, registrándose una actitud positiva hacia esta materia. Desde esta perspectiva, se encontró que la actitud del estudiantado universitario se resume de la manera siguiente:

La interacción ordenador y matemática es entendida como la vinculación del conocimiento, por ejemplo, forma de los gráficos y sus ecuaciones ( $M=4.16$; $S D=0.99$ ), coincidiendo con el estudio de Galbraith y Haines (2000), que afirman que los estudiantes mejoran su aprendizaje cuando interactúan con la computadora desde dos perspectiva: la gran cantidad de ejemplos que pueden manejar con las herramientas tecnológicas; y poder ampliar los datos que se presentan en la pantalla al hacer una búsqueda de la información, una vez terminada la sesión. La actitud hacia el aprendizaje de las matemáticas con tecnología es comprendida como el esfuerzo adicional del uso de las computadoras en matemática ( $M=3.81 ; S D=1.13$ ), así como dicen Pierce, Stacey y Barkatsas (2007) la tecnologia ofrecen nuevos enfoques para la enseñanza y por lo tanto para el aprendizaje dentro y fuera del aula.

Las actitudes del uso de la tecnología en el aprendizaje de las matemáticas son percibidas como el disponer de la tecnología para hacer el trabajo rutinario permite probar diferentes métodos y enfoques $(M=3.91 ; S D=1.01$ ) y el querer mejorar en el uso de las computadoras para que me ayude en las matemáticas ( $M=3.91$; $S D=1.16)$; según Cretchley (2007) estos resultados proporcionan los sentimientos de gusto y motivación hacia el uso de las tecnologías cuando se hace y se aprende matemáticas. Las experiencias matemáticas con tecnologías son apreciadas en términos generales como el aprender utilizar software para hacer matemáticas ( $M=3.62$; $S D=1.15)$, es decir, que la experiencia personal en el uso de softwares mejora sus actitudes hacia las matemáticas. Y finalmente, matemáticas y tecnologías es intuida por el estudiantado como el gusto de aprender matemáticas con ayuda de la computadora ( $M=3.60 ; S D=1.18)$; y que la retroalimentación inmediata de la computadora es útil para resolver problemas matemáticos ( $M=3.52 ; S D=1$.13), Según Nguyen y Kulm (2005), este gusto hacia la matemática y tecnología se manifiesta porque el profesorado diseña ambientes de aprendizajes con recursos tecnológicos accesibles y amistosos provocando el interés del estudiantado hacia las matemáticas.

En este sentido, las actitudes del estudiantado ante la incorporación de tecnologías en la enseñanza de las matemáticas también se pueden explicar desde el punto de vista de las relaciones entre las categorías hacia el uso de la tecnología en el aprendizaje de las matemáticas, es decir, que las experiencias matemáticas con tecnologías se asocian con matemáticas y tecnologías con una $R=0.674$, entendiéndose como el gusto por la integración de las tecnologías en matemáticas. Así también se encontró una relación entre las actitudes hacia el aprendizaje de las matemáticas con tecnológicas y experiencias matemáticas con tecnología con una $R=0.654$, manifestándose como el gusto y motivación del estudiantado hacia las matemáticas en su experiencia personal con el uso de software en el aprendizaje de esta asignatura. (Pierce, Stacey, \& Barkatsasb, 2007; Cretchley, 2007)

Por otra parte, se estudió si existen diferencias estadísticamente significativas entre género en relación con las categorías de actitudes hacia el uso de la tecnología en el aprendizaje de las matemáticas, encontrando que hombres y mujeres poseen las mismas actitudes hacia el proceso de enseñanza y aprendizaje de las matemáticas con tecnologías, ya que los valores de significancia p es mayorque 0.05 , estos resultados coinciden con los estudios de Meyer y Kohler (1990) y, Ursini y Sánchez Ruiz (2008) los cuales no encontraron diferencias significativas en las actitudes de hombres y mujeres cuando se usan las tecnologías en las clases de matemáticas. Resultados similares fueron encontrados por Kloosterman, Tassell, Ponniah y Essex (2001), concluyeron que los grupos de estudiantes universitarios, tanto de las zonas rurales como urbanas, acreditaban que, en general, las matemáticas no eran una cuestión de género, es decir, las matemáticas presentar un carácter neutral hacia al género.

Algo importante a mencionar son las diferencias estadísticamente significativas entre la variable edad en relación con las categorías de actitudes hacia el uso de la tecnología en el aprendizaje de las matemáticas encontrados que las actitudes hacia el aprendizaje de las matemáticas con tecnologías; experiencias matemáticas con tecnologías; y matemáticas y tecnologías las edades entre 17-18 y 19-20 poseen diferencias significativas a favor de la edad entre 19 y 20 lo que significa que ha mayor edad del estudiantado mejores actitudes hacia la incorporación de las matemáticas con tecnologías. Mientras que en 
la categoría la interacción ordenador y matemáticas; y actitudes del uso de la tecnología en el aprendizaje de las matemáticas, las edades entre 17 a 20 tienen el mismo grado actitudes hacia el aprendizaje de las matemáticas con tecnologías. En definitiva, la edad es un factor determinante en el aprendizaje de las matemáticas con tecnologías, porque el gusto y la motivación se manifiestas a tempranas edades y porque a mayor edad mejores experiencias hacia el uso y aprender matemáticas con tecnologías.

Finalmente, se estudio si existen diferencias estadísticamente significativas entre la variable especialidad en relación con las actitudes hacia el uso de la tecnología en el aprendizaje de las matemáticas obteniendo como resultado que, las especialidades poseen el mismo grado actitud hacia la incorporación de tecnologías en la enseñanza de las matemáticas, pero mejor posicionada la especialidad en informática. Estos resultados coinciden con el estudio de Flores y Auzmendi (2016), donde se mencionan que el estudiantado universitario tiene actitudes positivas hacia las matemáticas en actividades que involucren a los procesos matemáticos con tecnologías, lo que permite mayor confianza hacia la resolución de problemas. Así mismo, se relacionan con los estudios de Hidalgo, Maroto Sáenz y Palacios Picos (2005), Fernández Cézar y Aguirre Pérez (2010) y Cardoso Espinosa, Vanegas López y Cerecedo Mercado (2012), en el sentido que las actitudes hacia las matemáticas pueden considerarse como motor del aprendizaje, y que estas pueden influir en la decisión sobre futuras oportunidades de su carrera profesional.

\section{Conclusiones}

El objetivo central de esta investigación, ha sido analizar las actitudes del estudiantado ante la incorporación de tecnologías en la enseñanza de las matemáticas encontrando una actitud globalmente favorable, esto se debe al modelo de formación en matemáticas de la Universidad de las Regiones Autónomas de la Costa Caribe Nicaragüense que se sustenta en ambientes de aprendizajes que incorpora entornos informáticos como Maple, Matlab, Wrmaxima, Derive, Mathematics, GeoGebra, Wiris, R, SPSS y dispositivos tecnológicos como celulares con aplicaciones abiertas para calcular y graficar, así también calculadoras gráficas (Flores López \& López Mairena, 2016). Además, que promueve el aprendizaje colaborativo que ofrece la plataforma Moodle interactuando en foros, chat, cuestionarios, pruebas en líneas, talleres, scorm, glosarios, wiki, y audio-visuales y online. (Flores López \& Rojas Robles, 2013)

Se señala que, hombres y mujeres tienen el mismo grado de actitud, y que los estudios de género y matemáticas con incorporación de tecnologías en el aula de clases, también se ha vuelto cada vez más necesario precisar la concepción de género. Es decir, que el género es un sistema ideológico que, a través de distintos procesos, dirige las maneras diferenciadas en las que hombres y mujeres modelan su percepción del mundo y su rol en la sociedad. Este sistema ideológico dirige su percepción de las diferentes disciplinas escolares, en particular las matemáticas, y como se relacionan con ellas. Según Flores Palacios (2000), la conciencia de género se adquiere a través de procesos sociales complejos y se reproduce mediante una serie de factores como, por ejemplo, la educación, que tienen, entre otros, el propósito de mantener y trasmitir los valores culturales que dominan en cierta sociedad. En particular, coincidimos con los estudios de Sánchez Ruiz y Ursini (2010), porque señalan que la academia, institución encargada de educar, tiene entre sus funciones también la de reproducir y fortalecer las diferencias de género, y lo hace a través de sus normas y contenidos curriculares.
Se resalta que los estudiantes de edades entre 17 y 18 pueden utilizar con frecuencia más tecnologías básicas, pero que las habilidades que demuestran a la hora de utilizar las tecnologías para el que hacer y aprender matemáticas son muy limitadas, aunque, se destaca que los estudiantes entre 19 y 20 les gusta tener experiencias matemáticas con tecnologías porque mejora su aprendizaje. También, se señala que las especialidades en informática, ingeniería civil y matemática posee la misma actitud de aprender matemáticas con incorporación de tecnologías. Entonces, las tecnologías de la información y comunicación son herramientas mediadoras entre la percepción y aprendizaje del conocimiento matemático (Sandoval Caceres \& Moreno Armella, 2012). En este sentido, el profesorado debe apropiarse de la tecnología, el diseño y elaboración de secuencias didácticas significativas donde el estudiantado realicen trabajos de discusión y retroalimentación (Sánchez Medina, Cabrera Medina, \& Martinez Gaitan, 2016). Así mismo, decir que la integración de la plataforma web, teléfonos móviles, presentaciones PowerPoint; y correos electrónicos en la enseñanza contribuyen en el aprendizaje colaborativo. (Gonzalez Valencia, 2015)

Para finalizar, se recomienda la integración de las tecnologías en la práctica del profesor, es decir, en la enseñanza de las matemáticas, tomando en cuenta: que lo hace como un modo de mejorar las experiencias de aprendizaje del estudiantado (dimensión pedagógica); que conoce y maneja bien las tecnologías disponibles para apoyar su función docente (dimensión técnica e instrumental); que utiliza las tecnologías para mejorar la gestión curricular en su ámbito de acción (dimensión de gestión); que se sirve de las tecnologías como un medio de inclusión social, de atención a la diversidad, realizando una actuación ética y legal respecto de su uso y cuidando la salud y del medio ambiente (dimensión social, ética, y legal); y finalmente, que reconoce su responsabilidad para que el estudiantado tenga un aprendizaje cada vez más eficiente y actual, usando o incorporando las tecnologías y como docente asuma responsablemente su propia actualización y desarrollo profesional con las potencialidades que presentan las tecnologías para su quehacer profesional (dimensión responsabilidad y desarrollo profesional).

\section{Referencias}

Albert Gómez, M. J. (2007). La Investigación Educativa: Claves teóricas. Madrid [es]: McGraw-Hill.

Alvarez, Y., \& Ruíz Soler, M. (2010). Actitudes hacia las Matemáticas en Estudiantes de Ingeniería en las Universidades Autónomas Venezolanas. Revista de Pedagogía, 31(89), 225-249. Obtenido de http://www.scielo.org.ve/scielo. php? script $=$ sci arttext\&pid $=$ S0798-97922010000200002

Area Moreira, M., Gros Salvat, B., \& Marzal García-Quismondo, M. Á. (2008). Alfabetizaciones y Tecnologías de la Comunicación y la Información. Madrid [es]: Síntesis.

Auzmendi Escribano, E. (1992). Las Actitudes hacia la Matemática-Estadística en las Enseñanzas Medias y Universitarias. Bilbao [es]: Mensajero.

Balacheff, N., \& Kaput, J. J. (1996). Computer-Based Environments in Mathematics. En A. Bishop, M. A. Clements, C. Keitel-Kreidt, J. Kilpatric, \& C. Laborde (Edits.), International Handbook of Mathematics Education (págs. 469501). London [uk]: Wolkers Kluwer.

Bisquerra Alzina, R. (2009). Metodología de la Investigación Educativa. Madrid [es]: La Muralla.

Bolívar Ruiz, C. (2010). Actitudes de Profesores Universitarios hacia el Uso de Educativo de las Tic: Conceptualización y medición. Paradigma, 33(2) Obtenido de http://www.scielo.org.ve/scielo.php?script = sci_arttext\&pid = S1011-22512012000200002

Cardoso Espinosa, E. O., Vanegas López, E. A., \& Cerecedo Mercado, M. T. (2012) Diagnóstico sobre las Actitudes hacia las Matemáticas del Estudiantado que Inicia Sus Estudios en Tres Posgrados en Administración de Empresa. Educare, 16(2), 237-253. Obtenido de http://revistas.una.ac.cr/ index.php/EDUCARE/article/viewFile/4052/3890 


\section{Incorporación de tecnologías en la enseñanza de las matemáticas}

\section{Actitudes del estudiantado universitario}

Cretchley, P. (2007). Does Computer Confidence Relate to Levels of Achievement in ICT-Enriched Learning Models? Education and Information Technologies, 12(1), 29-39. doi:10.1007/s10639-006-9004-6

Depool Rivero, R. A. (2005). La Enseñanza y Aprendizaje del Cálculo Integral en un Entorno Computacional: Actitudes de los estudiantes hacia el uso de un programa de cálculo simbólico. Números, 62, 3-31. Obtenido de http://www.sinewton.org/numeros/index.php?view = weblink\&catid = 44\%3Avolumen-62\&id = 86\%3Ala-ensenanza-y-aprendizaje-del-calculointegral-en-un-entorno-computacional-actitudes-de-los-estudianteshacia-el-uso-de-un-programa-de-calculo-simbolico\&option =com weblinks

Dettori, G., Garuti, R., \& Lemut, E. (2001). From Arithmetic to Algebraic Thinking by Using a Spreadsheet. En R. Sutherland, T. Rojano, A. Bell, \& R. Lins (Edits.), Perspectives on school algebra (págs. 191-207). London [uk]: Wolkers Kluwer. doi:10.1007/0-306-47223-6

Fernández Cézar, R., \& Aguirre Pérez, C. (2010). Actitudes Iniciales hacia las Matemáticas de los Alumnos de Grado de Magisterio de Educación Primaria, Estudio de una Situación en el EEES. Unión, Revista Iberoamericana de Educación Matemáticas(23), 107-116.

Flores, W. O., \& Auzmendi Escribano, E. (2015). Análisis de la Estructura Factorial de una Escala de Actitud hacia las Matemáticas. Aula de Encuentro, 17(1), 45-77. Obtenido de https://revistaselectronicas.ujaen.es/index.php/ADE/ article/view/2256

Flores, W. O., \& Auzmendi Escribano, E. (2016). Análisis Ontosemiótico en los Procesos de Resolución de Problemas Matemáticos por Estudiantes Universitarios. Bilbao [es]: Universidad de Deusto.

Flores, W. O., \& López Mairena, E. (2016). Recursos Didácticos y Tecnológicos para la Enseñanza de la Integral Definida en el Modelo de Universidad Comunitaria Intercultural. Ciencia e Interculturalidad, 18(1), 63-78. doi:10.5377/rci.v18i1.3050

Flores, W. O., \& Rojas Robles, N. (2013). Integración de las Tecnologías de la Información y Comunicación en la Enseñanza Universitaria. Horizontes Pedagógicos, 15(1), 46-57. Obtenido de http://revistas.iberoamericana. edu.co/index.php/rhpedagogicos/article/view/404

Flores Palacios, F. (2000). Psicología Social y Género: El sexo como objeto de representación social. México D.F. [mx]: Universidad Nacional Autónoma de México.

Galbraith, P., \& Haines, C. (2000). Mathematics-Computing Attitudes Scales (Vol. Monographs in Continuing Education). London [uk]: University London.

Gil Flóres, J., Rodríguez Gómez, G., \& García Jiménez, E. (1995). Estadística Básica Aplicada a las Ciencias de la Educación (412 ed.). Sevilla [es]: Kronos.

Gil, N., Gil Blanco, L., \& Guerrero, E. (2005). El Dominio Afectivo en el Aprendizaje de las Matemáticas: Una revisión de sus descriptores básicos. Unión, Revista Iberoamericana de Educación Matemáticas(2), 15-32. Obtenido de http:// www.fisem.org/www/union/revistas/2005/2/Union_002_004.pdf

Gómez Chacón, I. M. (2009). Actitudes Matemáticas: Propuesta de transición del bachillerato a la universidad. Educación Matemática, 21(3), 5-32. Obtenido de $h t t p: / / w w w$. scielo.org. $m x /$ scielo.php? script = sci arttext\&pid = S1665-58262009000300002

Gómez Chacón, I. M., \& Haines, C. (2008). Students' Attitudes to Mathematics and Technology: Comparative study between the United Kingdom and Spain. 11th International Congress on Mathematical Education [ICME-11]. Monterrey [mx]: ICME-11.

Gonzalez Valencia, H. (2015). La Integración de la Tecnología como Herramienta Significativa en la Enseñanza del Inglés como Lengua Extranjera. Horizontes Pedagógicos, 17(1), 53-66. Obtenido de http://revistas. iberoamericana.edu.co/index.php/rhpedagogicos/article/view/651

Hernández Sampieri, R., Fernández Collado, C., \& Baptista Lucio, P. (2010). Metodología de la Investigación (600 ed.). México D.F. [mx]: McGraw-Hill.

Hidalgo Alonso, S., Maroto Sáenz, A., \& Palacios Picos, A. (2005). El Perfi Emocional Matemático como Predictor de Rechazo Escolar: Relación con las destrezas y los conocimientos desde una perspectiva evolutiva. Educación Matemática, 17(2), 89-116. Obtenido de https:// www.researchgate.net/publication/237769264_El perfil_emocional matematico como predictor de rechazo escolar relacion con las destrezas y los conocimientos desde una perspectiva evolutiva

Hoyles, C., \& Sutherland, R. (1989). Logo Mathematics in the Classroom (238 ed.). London [uk]: Routledge
Kloosterman, P., Tassell, J. H., Ponniah, A. G., \& Essex, N. K. (2001). Mathematics as a Gendered Domain in the United States. [interactive symposium] Mathematics: Still a Male Domain?, Seatle. 13, págs. 1-15. Seatle [us]: AERA, The American Educational Research Association. Obtenido de http://citeseerx.ist.psu.edu/viewdoc/download?doi = 10.1.1.22.5640\&rep $=$ repl\&type $=p d f$

López, R., Castro Martínez, E., \& Molina, M. (2013). Actitudes de Estudiantes de Ingeniería de Nuevo Ingreso hacia el Uso de la Tecnología en Matemáticas. PNA, 8(1), 31-50.

Mariotti, M. (2005). New Artefacts and Mathematical Meanings in the Classroom. En R. Sutherland, \& F. Olivero (Edits.), Visions of mathematics education: Embedding technology in learning (pág. 579). Bristol [uk]: University of Bristol; Department of Social Medicine Health Care Evaluation Unit.

Martínez Padrón, O. J. (2008). Actitud hacia las Matemáticas. Sapiens, 9(1), $237-$ 256. Obtenido de https://dialnet.unirioja.es/descarga/articulo/2781941.pdf

McLeod, D. B., \&Adams, V. M. (1989). Affect and Mathematical Problem Solving: A new perspective (268 ed.). New York [us]: Springer-Verlag. doi:10.1007/978$1-4612-3614-6$

Meyer, M., \& Koehler, M. (1990). Internal Influences on Gender Differences in Mathematics. En E. Fennema, \& G. C. Leder, Mathematics and Gender (págs. 60-95 [214]). New York [us]: Columbia University.

Nguyen, D. M., \& Kulm, G. (2005). Using Web-Based Practice to Enhance Mathematics Learning and Achievement. Journal of Interactive Onlive Learning, 3(3), 16. Obtenido de https://pdfs.semanticscholar.org/a6b7/ aa65fe63e0510c2e89ad66f649b36fcd1975.pdf

Pérez Tyteca, P., Castro Martínez, E., Rico Romero, L., \& Castro Martínez, E. (2011). Ansiedad Matemática: Género y ramas de conocimiento en alumnos universitarios. Enseñanza de las Ciencias, 29(2), 237-250. Obtenido de http://www.raco.cat/index.php/Ensenanza/article/view/243835

Petriz Mayen, M. A., Barona Ríos, C., López Villareal, R. M., \& Quiroz González, J. (2010). Niveles de Desempeño y Actitudes hacia las Matemáticas en Estudiantes de la Licenciatura en Administración en un Universidad Estatal Mexicana. Revista Mexicana de Investigación Educativa, 15(47), 1223-1249. Obtenido de http://www.scielo.org.mx/scielo.php?script = sci arttext\&pid $=$ S1405-66662010000400012

Pierce, R. (2002). An Exploration of Algebraic Insight and Effective Use of Computer Algebra Systems. Melbourne [au]: Universidad de Melbourne.

Pierce, R., Stacey, K., \& Barkatsasb, A. (2007). A Scale for Monitoring Students' Attitudes to Learning Mathematics with Technology. Computers \& Education, 48(2), 285-300. doi:10.1016/j.compedu.2005.01.006

Sabariego Puig, M. (2004). El Proceso de Investigación. En Metodología de la investigación educativa (págs. 127-163). Madrid [es]: La Muralla.

Sánchez Medina, I. I., Cabrera Medina, J. M., \& Martinez Gaitan, J. E. (2016). Ayudas Virtuales como Apoyo al Aprendizaje Inclusivo en la Ingeniería. Horizontes Pedagógicos, 18(1), 81-95. Obtenido de http://revistas. iberoamericana.edu.co/index.php/rhpedagogicos/article/view/839

Sánchez Ruiz, J. G., \& Ursini, S. (2010). Actitudes hacia las Matemáticas y Matemáticas con Tecnología: Estudios de género con estudiantes de secundaria. Revista Latinoamericana de Investigación en Matemática Educativa, 13(4), 303-318. Obtenido de http://www.clame.org.mx/ relime/201017d.pdf

Sánchez Ruiz, J. G., Orendain, M., \& Ursini, S. (2004). Validación y Confiabilidad de una Escala de Actitudes hacia las Matemáticas y hacia las Matemáticas Enseñadas con Computadora. Educación Matemática, 16(3), 59-78. Obtenido de http://www.redalyc.org/pdf/335/33529137018.pdf

Sandoval Caceres, I. T., \& Moreno Armella, L. E. (2012). Tecnología Digital y Cognición Matemática: Retos para la educación. Horizontes Pedagógicos, 14(1), 21-29. Obtenido de http://revistas.iberoamericana. edu.co/index.php/rhpedagogicos/article/view/109

Ursini, S., \& Sánchez Ruiz, J. G. (2008). Gender, Technology and Attitude Towards Mathematics: A comparative longitudinal study with Mexican students. ZDM Mathematics Education, 40(4), 559-577. doi:10.1007/s11858-0080120-1

Vale, C. M. (2001). Gender and Computer-Based Mathematics in Selected Secondary Classrooms. Victoria [au]: La Trobe University.

Volman, M., \& van Eck, E. (2001). Gender Equity and Information Technology in Education: The second decade. Review of Educational Research, 71(4), 613-634. Obtenido de https://www.jstor.org/stable/3516100 